\title{
A Coordinated European Effort for the Definition of a Satellite Integrated Environment for Future Mobile Communications
}

\author{
Enrico Del Re, University of Florence
}

$T$ hird-generation systems for mobile and personal communications are under study worldwide; standardization bodies in Europe - such as the European Telecommunications Standardization Institute (ETSI) - and worldwide - that is, the International Telecommunications Union - Radio (ITU-R) - are currently working on defining the specifications of systems for the beginning of the next century. The Universal Mobile Telecommunications System (UMTS) is the name given by ETSI to the new-generation system, and the Future Public Land Mobile Telecommunications System (FPLMTS) is the name used by ITU. Their concepts, schematically illustrated in Figs. 1 and 2, are very similar and aim to provide a wide variety of services (possibly the same set of services as the fixed network) to mobile users with the same quality as that offered by the fixed network (i.e., the integrated services digital network - ISDN). It is an operational requirement that UMTS and FPLMTS users will be able to use universal personal telecommunications (UPT) services from their terminals; that is, they will provide both terminal and personal mobility. The necessary functionality can be located within UMTS-FPLMTS or associated intelligent fixed networks or intelligent terminals, or distributed among them. Clearly, this functionality will enable the intelligent network to provide communication services to both mobile and fixed means of access freely and in a logically consistent way.

Since the early stage of UMTS and FPLMTS definition, it has been recognized that the satellite component should be an essential part of the overall system and that the two components (terrestrial and satellite) must be as closely integrated as possible. This is in contrast with most of the terrestrial and satellite systems presently proposed by the industries, which were conceived separately so that no or only slight cooperation is possible between the terrestrial and satellite components, although the concept of satellite/terrestrial integration for mobile communications emerged in the late ' $80 \mathrm{~s}[1,2]$. On the contrary, the European COST 227 Integrated Space/Terrestrial Mobile Networks project promoted a coordinated effort for the definition of a satellite system integrated as closely as possible with the terrestrial part. In the following we

This work has been supported by the Italian Space Agency and MURST. summarize the objective and results of this project, begun in 1991 and ended in 1995.

This project set up fruitful cooperation and exchange of information and results with other related European research projects; in particular, RACE SAINT (Satellite Integration in the Future Mobile Network) and COST 231, "Evolution of Land Mobile Radio (Including Personal) Communications," and standardization bodies like ETSI. The effect of this cooperation was to avoid duplication of effort and, more important, to identify and evaluate those technical solutions more appropriate for both environments (terrestrial and satellite).

\section{THE ROLE OF SATELLITE IN AN INTEGRATED ENVIRONMENT}

The meaningful characteristics of a satellite system capable of supporting future terrestrial systems in mobile applications are the following:

Complete Coverage - The complete deployment of a terrestrial mobile network takes several years due to the network infrastructures needed to cover the entire service area. As a consequence, such deployment will start in more densely populated geographical (metropolitan) areas, where the communication demand is higher, whereas areas characterized by spread-out population and/or low communication demand (rural and developing areas) will have to wait a long time for a homogeneous distribution of the network facilities. In this development phase a cellular system needs support from the satellite system, able to connect all those locations not yet covered. Complete coverage of the service area can be provided with cooperation between the two systems.

Coverage Extension - Another interesting feature of an integrated system (terrestrial-satellite) is the possibility of extending coverage to users outside the planned service area of the cellular system. In this way, for example, European coverage could be extended to Eastern Europe, part of North Africa, and the Middle East. Moreover, it is worth highlighting that the application fields for a mobile satellite system (MSS) can be different from those of traditional land mobile 
services. For example, maritime and aeronautical users can be connected, by means of satellite systems, with each other and with terrestrial mobile users.

Dynamic Traffic Management As is well known, the most meaningful characteristic of a satellite system is its intrinsic flexibility. This aspect is particularly useful in a mobile environment, where the subscribers' mobility introduces additional randomness to the traffic offered to the system. Overload situations can easily be solved if dynamic (reconfigurable) channel allocation is forecast for the system. This feature is more useful when spot-beam coverage is adopted, and processing and routing capabilities are present onboard.

\section{"Disaster-Proof" Link Availabili-}

ty - A further advantage of an

integrated system can be seen in cases of disasters, earthquakes, and in any situation in which a terrestrial network is highly vulnerable. In fact, in these cases the satellite could represent the only communication available to the local population and rescue organizations.

On the basis of these considerations, and taking into account that a satellite system can represent an alternative choice for traffic routing, increasing the global network efficiency, a high level of integration between the two systems is desirable. In particular, a key point of such integration could be the user's capability of operating with the same terminal. Nevertheless, it should be noted that the integration of a satellite system with a terrestrial one involves a lot of problems, such as:

- Design of the overall architecture

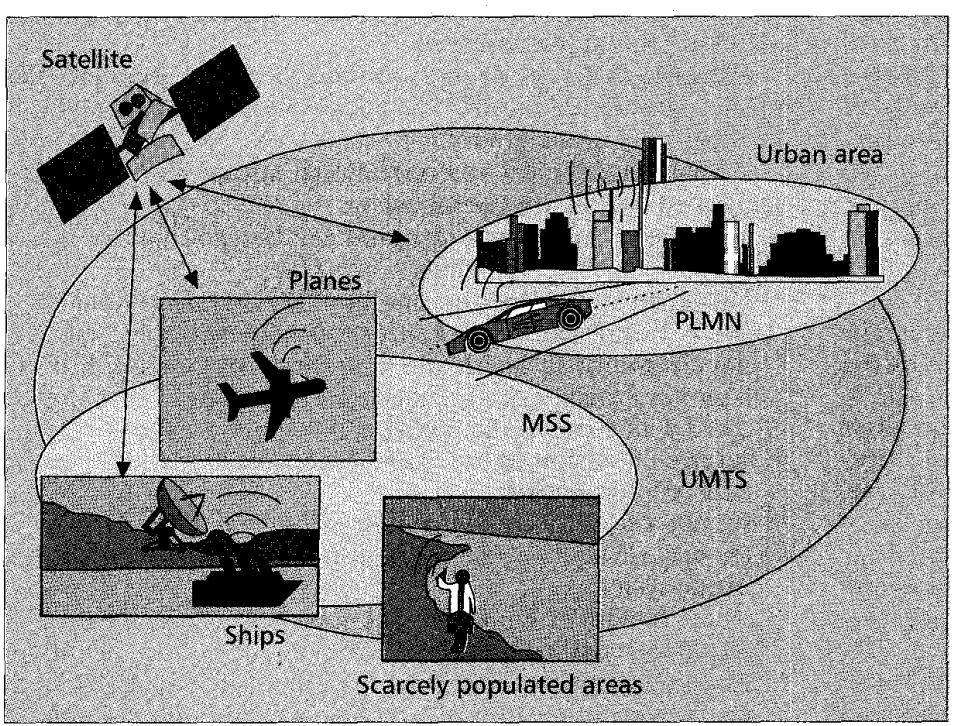

Figure 2. Logical (simplified) architecture of UMTS.
- Call handling and routing

- Signaling and communication protocols

- Network interfaces

- Procedures of mobile localization

- Handover procedures between the two systems

- Systems synchronization

- Frequency coordination

- Transmission and access protocols

The achievable level of integration depends on the solution proposed for each of them.

Anyway, several levels of integration are possible. In the following, the traditional classification in the literature is reported.

Geographical Integration - In this case, rather than speaking of integration, it would be better to say that the satellite system complements the terrestrial one, its main objective being to offer communications services to areas not served via ground infrastructures. The two networks are independently conceived, so they are based on different technologies and, in general, offer different services to users. This means that in this case the generic user has to choose to buy either a "terrestrial" or "satellite" terminal or, as an alternative, a dualmode terminal able to switch from one to the other. Moreover, the kind of terminal owned by each user must be known by a fixed user originating the call, in order to correctly route the call via the terrestrial or satellite network.

Services Integration - In this case, the two networks can also be based on different technologies, still distinct. The substantial difference, with respect to the geographical level of integration, resides in the fact that the satellite system parameters are now chosen to support services compatible with those provided by the cellular system, in this case with a lower service quality. This means that the same mobile terminal is able to access both systems. It is sufficient, in fact, 


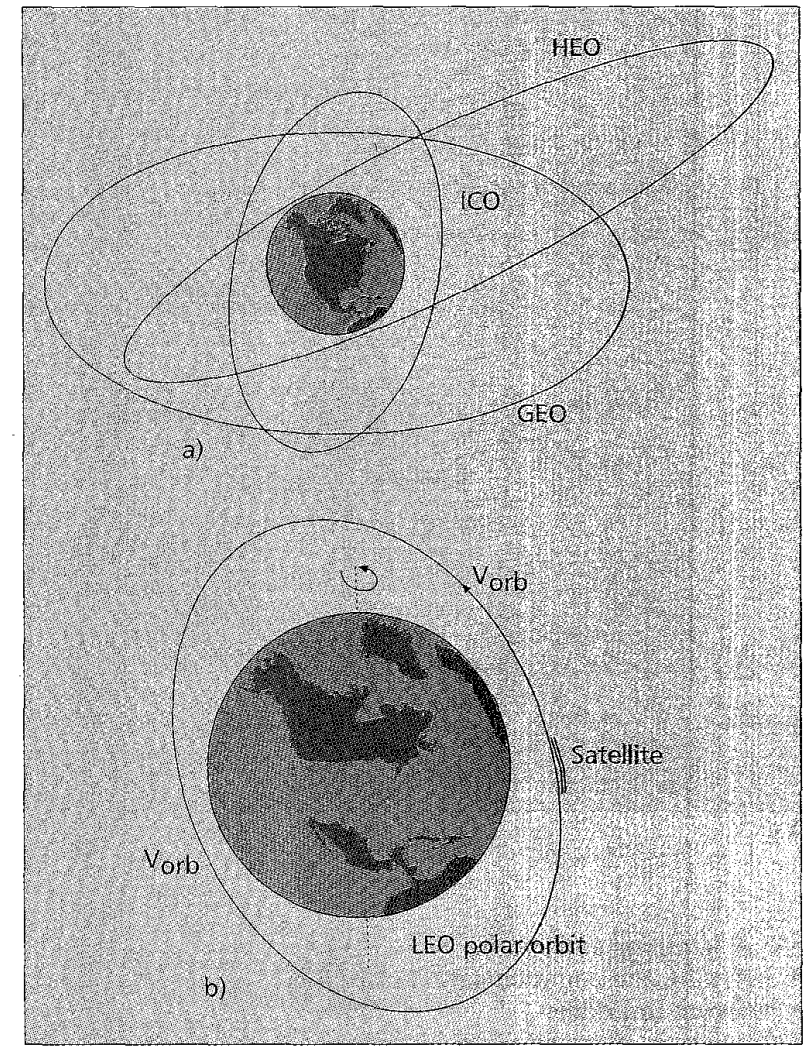

Figure 3. a) Illustrative example of GEO, $H E O$ and ICO orbits; b) Illustrative example of $L E O$ orbit.

that the terminal be able to provide appropriate protocol conversions.

Network Integration - This integration level is characterized by network infrastructures common to both systems. It should be noted that this approach does not prevent designing the satellite system with rigorous compatibility constraints imposed on the terrestrial system. On the contrary, it can still be based on techniques and system parameters optimized for satellite applications. Just to clarify, the advantage of this type of integration is easier service utilization for mobile subscribers. Common network infrastructures, in fact, allow a fixed user to ask for a connection with a mobile user without worrying about call routing (via a terrestrial or satellite network) or knowing which kind of terminal is owned by the mobile user. A unique calling number identifies the generic mobile subscriber in this case, and the mentioned actions are handled by the network managing units.

Technique Integration - The only, though substantial, difference of this approach from the previous one concerns the techniques characterizing the satellite system. In this case, in fact, the same (or at least as similar as possible) techniques as those of the terrestrial system, in terms of access scheme, protocols, bit rates, and so on, are adopted for the satellite one. The advantage of such a solution is a considerable reuse of the cellular technologies for implementation of the dual-mode mobile terminal. This could in fact utilize common baseband protocol and modulation equipment for both the operating modes, whereas due to the different frequency bands characterizing the terrestrial and satellite systems, duplication of some equipment, such as radio frequency (RF) circuitry and ad hoc antenna, is necessary.
System Integration - According to the last integration level, the satellite is no longer seen as alternative routing able to support communications in geographical areas not covered by the terrestrial system, but, finally, as part of a unique really integrated system. According to this solution, handover of calls in progress between terrestrial and satellite cells could be realized each time it becomes necessary due to partial channel occupancy, degradation of some links, and so on. It must be noted, however, that rerouting procedures can be performed only if the mobile user interested in this is equipped with a dual-mode terminal.

To conclude this section, it is worth underlining that each level of integration is characterized by different critical areas, which become more numerous when higher levels are considered. As to the system integration level, for example, problems related to "system" handover procedures and synchronization protocols, the different link characteristics of the two systems, the maximum compatibility between all aspects of the systems, and so forth have to be deeply investigated in order to propose a successful integrated system.

\section{COSt 227 StATUS AND ObJECTIVES}

The COST 227 Integrated Space/Terrestrial Mobile Networks project started in April 1991 and coordinated the research of 23 teams from industry, operators, research centers, and universities from 12 European countries (Belgium, Czech Republic, France, Hungary, Germany, Greece, Italy, Poland, Portugal, Slovenia, Switzerland, and United Kingdom) and the European Space Agency. It has been organized in three Working Groups (WGs):

WG1: $\quad$ Satellite System Architecture

WG2: Network Aspects

WG3: Radio Interface

The objectives were to study and define feasible systems for mobile communications based on the integration of a satellite network and a terrestrial network, where the integration should aim at:

- Providing, as far as possible, the largest set of common services

- Identifying the largest possible set of common functions at the mobile terminal

- Identifying the largest possible set of common functions at the ground infrastructure

Because different degrees of integration are possible, the project considered feasible technical solutions for the highest possible level of integration.

The main technical and operational advantages of such an integrated system are the following:

- A low-cost dual-mode terminal can be used due to the reuse of common functions.

- The earth stations of the satellite netwotk are similar to the cellular switching centers: this avoids expensive development of earth stations which can be adapted from terrestrial cellular stations and may even be collocated with some of them, saving the common parts.

- Service extension to areas not yet covered by the terrestrial cellular network.

- Service extension to aeronautical and maritime mobiles (in agreement with the concept of a personal communication system).

- Better quality of service for the user in the areas covered by the terrestrial cellular network, due to the backup function of the satellite link in case of unavailability of the terrestrial resources (traffic congestion, link failures, emergency events, maintenance, etc.) 


\section{MAIN RESULTS}

n the following we shall summarize the main results and preliminary proposals obtained by each WG. These results are extracted from the documents produced by the project and are more detailed in the Final Report [3].

\section{WORKING GROUP 1}

Orbit Selection - The applicable orbits have been considered in detail. The following types of orbits were evaluated (Figs. $3 a$ and $3 b$ ):

- Geostationary orbit (GEO)

- Low-earth orbit (LEO)

- Intermediate circular orbit (ICO)

- Highly elliptical orbit (HEO)

Once the types of services are defined, the analysis of the system architecture implies the selection of suitable orbit configurations in terms of constellation characteristics, coverage area, service availability, and handover aspects.

Once a certain continuity degree of service is defined, the chosen orbit influences the dimension of the satellite configurations and, consequently, the launch and positioning procedures of each satellite unit of the constellation. The major aspects of each orbit family and the related advantages and disadvantages are summarized in order to provide meaningful elements for comparison.

\section{GEO Orbit}

Main advantages:

- Simplicity of the satellite configuration; able to provide, with only three satellite units, continuous coverage up to $75^{\circ}$ latitude

- Well-known maintenance and control aspects of the GEO orbit

- Doppler effects of minor impact

- Spacecraft tracking not necessary for the user terminal

- Numerous launch opportunities Main disadvantages:

- Considerable signal delay due to the satellite-earth distance (about $125 \mathrm{~ms}$ for each link)

- More expensive class of launchers to limit the propellant for the orbital location maneuvers

- Low elevation angle on ground for latitude greater than $50^{\circ}$, which means higher atmospheric path losses and multipath and shadowing fading

\section{HEO Orbit}

Main advantages:

- Excellent view angles without obstructions (greater than $50^{\circ}$ everywhere in Europe); a good elevation also reduces the atmospheric path.

- High visibility period of each satellite (for the Molniya and Tundra families the visibility interval is about 6 and $12 \mathrm{~h}$, respectively).

Main disadvantages:

- Depending on the configuration, in any case a constellation of satellites must be considered to provide continuity coverage on a regional scale. An increased number of flight units must be generally expected with respect to the GEO case.

- In the visibility period each satellite is placed at a distance comparable to the GEO one.

- Handover procedures are necessary between satellites.

- The satellite motion determines slant range variation, Doppler effects, and variation of the antenna footprint

on the ground. Doppler compensation, power control, and suitable satellite antenna configuration have to be performed.

- Spacecraft tracking can be necessary for the user terminal.

- The class of launchers is the same as that of the GEO.

\section{LEO Orbit}

Main advantages:

- The satellites are orbited near to the earth (in the range $500-2000 \mathrm{~km}$ ), minimizing the time delay effects.

- The low orbit altitude allows considerably reducing the power requirements onboard and on the ground.

- A lower class of launchers is needed than in the GEO and HEO cases.

Main disadvantages:

- Many flight units are needed in order to provide coverage requirements in terms of elevation and continuity in the service area.

- The satellite constellation can require complex orbital design (each satellite must be suitably phased) and sophisticated orbital maintenance.

- It can be difficult to minimize the satellite number to cope with continuous (or quasi continuous) services on a regional scale.

- The visibility period of each satellite (some minutes) requires frequent handover activations.

- The slant range towards the same user drastically changes during the satellite pass, requiring a suitable antenna gain configuration onboard.

- Spacecraft tracking can be necessary for the user terminal.

- Doppler effects.

\section{ICO Orbit}

Ranging the orbit between 10,000 and $14,000 \mathrm{~km}$ from the Earth's surface, the characteristics (advantages and disadvantages) of this satellite constellation are between those of the GEO and LEO cases.

In nongeostationary orbit (NGSO) two general types of satellite system architecture can be identified (Figs. 4 and 5). In the first one (GLOBALSTAR-like), the mobile station (MS) is connected to the nearest fixed earth station (FES), and the fixed network (in particular, the public switched telephone network - PSTN) is used to route the call to its destination (mobile, as in Fig. 4, or fixed). In the second one (IRIDIUM-like), the MS call is routed to its destination (again mobile, as in Fig. 5, or fixed) through a real space net- 


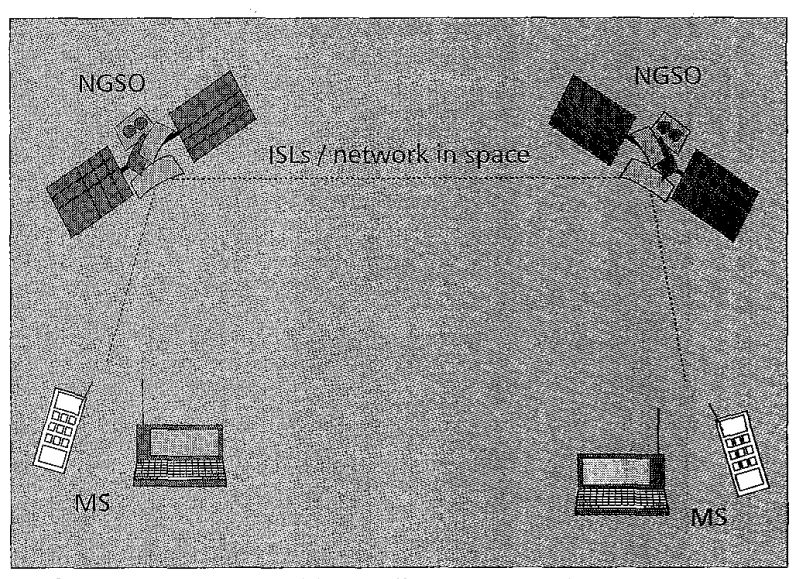

Figure 5. IRIDIUM-like satellite system architecture.

work of the satellites interconnected by intersatellite links (ISLs).

The applicability and choice of all the possible alternatives strongly depend on the identified service area.

For extended coverage of Europe, GEO and HEO configurations seem more suitable, due to the high inefficiencies and utilization costs characterizing the LEO and ICO systems in this case. For global coverage, on the contrary, LEO and ICO solutions could be effectively utilized and can be more suitable.

With regard to the HEO orbits, two systems have been analyzed. The first, concerning the M-HEO system concept, provides (multi)regional continuous coverage of the northern hemisphere; the second describes the Loopus orbits' characteristics; in both cases, the related pros and cons are outlined.

With regard to the ICO orbits, moreover, contributions have been presented aimed at describing the global mobile satellite system, named MAGSS-14, defined to provide personal communications at L-band.

Finally, a comparative analysis of the possible orbital configurations in terms of associated performance (elevation range, delays, handover frequency, etc.) are presently in progress, based on simulation runs. In particular, a new ICO configuration, named JOCOS, has been presented; it is capable of covering most of the earth, with seven satellites and an elevation angle better than $25^{\circ}$ from the mobile user.

Frequency Band - These activities were focused on the results of the World Administrative Radio Conference (WARC '92) held in Spain from February 3 to March 3, 1992.

All the revisions to the Radio Regulations were presented, particularly highlighting the new frequency allocations and sharing criteria and the modifications to the old ones.

The resulting problematic frequency coordination has been pointed out. According to the Conference results, the L, S and $\mathrm{Ka}$ bands were suggested as candidate frequencies from all the available ones. In particular, the $\mathrm{L}$ and $\mathrm{S}$ bands seem more suitable for LEO and ICO systems, whereas all three bands can be considered and tested for the other solutions.

\section{WORKING GROUP 2}

Research efforts are concentrated on the definition and analysis of different integrated network architectures.

Two network segments have been identified, the ground segment (GS) and the space segment (SS), and they are highly dependent on each other. The effects of the altitude on satellite constellation design and network aspects like handover statistics are of fundamental importance. Through the use of constellation simulation software, the basic groundwork has been lain from which more detailed networking studies can be launched.

Other studies have focused on the analysis of connectivity requirements for communication networks based on LEO satellites.

The Role of the Satellite - One of the important topics addressed in this activity is the priority level of the satellite resources. Two criteria can be defined:

- Satellite and terrestrial resources have the same priority, and the choice between them is based on a link quality criterion (satellite resources as purely additional ones).

- Satellite resources are considered more precious than the terrestrial ones and chosen only if terrestrial resources are not available for whatever reason (e.g. lack of coverage, congestion of channels, etc.) (satellite resources as second choice).

They represent a link-based and a network-based criterion, respectively. The project found that a more efficient resource management of the integrated system is obtained by the latter. Therefore, this criterion has been selected.

Resource Assignment - An important topic recently developed has been a comparison of the dynamic channel allocation (DCA) and fixed channel allocation (FCA) techniques. Comparing the call blocking probability of both techniques found that there was a significant advantage in using DCA. Moreover, the proposed DCA technique reduces the blocking probability for handover attempts with respect to new call attempts thanks to the used channel deallocation technique (channel rearrangement).

Preliminary considerations to privileged handover service with respect to call attempt service have been developed through simulations for both LEO and ICO solutions.

Mobility Management - Analytical models and derived traffic models for LEO systems, useful for providing design and performance evaluation of a LEO satellite network, have been taken into consideration. A comparison between LEO and ICO systems has been performed in terms of intersatellite handover. A code-division multiple access (CDMA) soft handover technique has been proposed to minimize the call dropping probability due to unsuccessful handover between LEO satellites.

A particular mobility management technique in a global personal communication system using resonant satellites constellation, such as MAGSS-14, has been evaluated.

Some basic problems of handover, different handover scenarios, and relevant signaling aspects have been considered when GSM and MSS are integrated in the same communication system. A substantial evolution of GSM mobility management techniques is required when integration with MSS using a dynamic satellite constellation is considered.

\section{WORKING GROUP 3}

Results include:

- The performance assessment of the TCH GSM channel in a satellite radio link, in which the performance of the GSM speech channel at "full" and "half" rates are evaluated for different propagation conditions and vehicle speeds. From the results it was concluded that the bit error rate (BER) performance is better at half than at full rate, but it depends very much on the reception of the multipath component.

- The study of a new class of codes, which can provide a powerful error correction for satellite systems with a very. efficient use of the hardware. 
- A study of CPM signals in selective fading and Gaussian noise channels with application to mobile-satellite channels.

- Modeling, simulation, and propagation measurements of mobile satellite-channels at the $\mathrm{L}, \mathrm{S}, \mathrm{Ku}$, and $\mathrm{Ka}$ bands have been performed.

- Efficiency comparison of multiple access systems for terrestrial cellular and satellite networks for a Gaussian channel: CDMA techniques perform better than frequencydivision multiple access (FDMA) and time-division multiple access (TDMA) and better than packet reservation multiple access (PRMA) in the presence of powerful coding; satellite networks are less efficient than their terrestrial counterparts, due to the diversity of the path attenuation and satellite antenna discrimination; CDMA satellite systems may have higher efficiency if polarization discrimination is used; and finally, better efficiencies could be achieved with regenerating satellites than with transparent ones.

- CDMA is a good candidate for a global communication network, and coherent reception has been analyzed by exploiting a pilot signal transmitted from the base station to estimate the channel impulse response.

- A method based on linear fractional spaced equalization (LFSE) for adaptively canceling the multiple access interference in CDMA systems offers significant gains over a conventional detector, eliminating the near-far problem without the explicit knowledge of the interfering spreading sequences; LFSE performs despreading and multipath combining in an easier way than a RAKE receiver.

- Analysis and simulation of the signal shape, power spectrum, and constellation diagram of new minimum shift keying (MSK)-type modulation techniques; namely, quadrature minimum shift keying (QMSK), multi-amplitude minimum shift keying (MAMSK), and quadrature multi-amplitude minimum shift keying (QMAMSK) show that QMAMSK has spectral efficiency better than MSK.

- The evaluation of a suitable separation distance between Radio Astronomy observation sites and MSS mobile terminals in order to avoid the interference problems arising from the shared use of bands has been performed.

The main future activities that deserve further investigations are in the areas discussed below.

Orbit Selection - Comparison and tradeoffs among LEO and ICO orbits will be assessed in terms of integration.

Type of Coverage - This activity will indicate the number of beams and the frequency reuse to obtain the required coverage and quality of service.

Resource Assignment - It is planned to investigate the performance of the novel proposed dynamic channel allocation (DCA) technique when different policies to handle the handover and new call requests are considered. The performance of the DCA technique will be compared with that obtained by using a fixed assignment approach.

Mobility Management - The localization, registration, and handover problems involved with an integrated terrestrial- satellite cellular network environment will be considered in detail, including the call setup procedures. In particular, the impact on mobility management techniques of nongeostationary satellite constellations will be taken into consideration.

Modulation, Coding, and Equalization - More suitable modulation, coding, and equalization techniques and related receivers, including those for CDMA systems, will be considered and analyzed. The comparison of all the techniques will be carried out taking into account the characteristics of the mobile channel.

Channel Characteristics - A suitable model for the satellite channel for mobile communication will be defined for the frequency band of interest. This model will be based on experimental propagation data (if available) or on theoretical and simulation work.

Multiple Access Technique - Basically, two multiple access techniques will be considered: TDMA and CDMA. Performance comparisons and trade-offs of these techniques will be carried out. An integrated system should perhaps have the same access technique for both the satellite and terrestrial networks. This activity is expected to give answers to this problem. In particular, the CDMA technique is presently of interest for both satellite and terrestrial links.

Biological Constraints - Provide state-of-the-art information about safety issues and some simulations on satellite applications.

\section{CONCLUSIONS}

$T_{\mathrm{r}}^{\mathrm{h}}$ e complete integration of a satellite network with a terrestrial cellular network is a system architecture challenge that requires solving problems at both the transmission and network levels. However, the potential advantages of the integrated system suggest careful study of the appropriate solutions.

The main technical advantages are:

- A limited increase in the complexity of the terminal for both the satellite and terrestrial systems, making it attractive for its potential low cost.

- The fixed earth stations (FESs) of the satellite system are similar to cellular base stations and switching centers; this avoids expensive development of FESs, which can be adapted from the terrestrial stations and may even be collocated with some of them, saving the common parts.

The main operational benefits that could be achieved are the extension of the mobile services (bearer services, teleservices, supplementary services) offered by the terrestrial system to:

- Not yet covered areas

- Eastern Europe and North Africa

- Aeronautical and maritime mobiles, so that one integrated land/aeronautical/maritime (LAM) system could be conceived for all mobiles

The definition of an integrated satellite/terrestrial mobile system is under study worldwide and for interesting comparison the reader is referred to $[4,5]$, where meaningful results from COST 227 and international research are reported.

Of course, the ultimate effective solution to the integrated 
satellite/terrestrial mobile system is still to be reached and requires additional in-depth studies, simulations and tests. To contribute to these activities a new project, COST 252, "Evolution of Satellite Personal Communications from 2nd to Future Generation Systems," has been proposed within the European Union, which, based on and starting from the results achieved in COST 227 and the

RACE Projects MONET and SAINT, also approaches an evolutionary migration of second-generation mobile systems (like GSM) towards future-generation personal systems, in order to save the large investments already made in present systems. We are looking forward to this and hope that the new COST 252 will become an operational research framework in a short time.

\section{ACKNOWLEDGMENT}

The results reported here are due to the research activities of the participants in COST 227. The cooperation, meetings, and discussions with them during the last four years represented a professional stimulus and personal pleasure for the author. He wishes to thank all COST participants whose dedicated and enthusiastic efforts produced valuable results and made the project management an easy and pleasant task.

\section{REFERENCES}

[1] E. Del Re, "Satellite System Integrated with the Terrestrial Cellular Network for Mobile Communications," European Space Agency Report, STR 228, Aug. 1989.

[2] E. Del Re, "A Satellite Mobile System Integrated with the Terrestrial Cellular Network," Proc. IEEE Int'l. Conf. on Commuñ. June 1989.

[3] "Integrated Space/Terrestrial Mobile Networks," COST 227 Final Report. European Union, Brussels, Belgium April 1995.

[4] IEEE JSAC, Special Issue on Mobile Satellite Communications for Seamless PCS, Feb. 1995.

[5] E. Del Re, Ed., "Mobile and Personal Communications," Proc. 2nd Joint COST 227/231 Workshop. [Elsevier Science, 1995].

\section{BIOGRAPHY}

ENRICO DEL RE [SM] received the Dr.Ing. degree in electronics engineering from the University of Pisa, Italy, in 1971. Since 1975 he has been with the Department of Electronics Engineering at the University of Florence, Italy, first as a research assistant, then as an associate professor, and since 1.986 as professor. During the academic year $1987-88$, he was on leave from the University of Florence for a nine-month period of research at the European Space Research and Technology Centre of the European Space Agency. The Netherlands. He is the co-editor of the book Satellite Integrated Communications Networks (North-Holland, 1988) and one of the authors of the book Data Compression and Error Control Techniques with Applications (Academic, 1985). He has been the chairman of the European Project COST 227, "Integrated Space/Terrestrial Mobile Networks." He received the $1988 / 89$ premium from the IEF (U.K.) for the paper "Multicarrier Networks." He received the $1988 / 89$ premium from the IEE (U.K.) for the paper "Multicarrier Demodulator for Digital Satellite Communication Systems:" 